Technical note

\title{
Generalized SDNR analysis based on signal and noise power
}

\author{
P. Monnin ${ }^{\mathrm{a}, *}$, S. Gnesin ${ }^{\mathrm{a}}$, F.R. Verdun ${ }^{\mathrm{a}}$, N.W. Marshall ${ }^{\mathrm{b}, \mathrm{c}}$ \\ a Institute of Radiation Physics (IRA), Lausanne University Hospital (CHUV) and University of Lausanne, Rue du Grand-Pré 1, 1007 Lausanne, Switzerland \\ ${ }^{\mathrm{b}}$ UZ Gasthuisberg, Department of Radiology, Herestraat 49, 3000 Leuven, Belgium \\ ${ }^{\mathrm{c}}$ Medical Imaging Research Center, Medical Physics and Quality Assessment, Katholieke Universiteit Leuven, 3000 Leuven, Belgium
}

\section{A R T I C L E I N F O}

\section{Keywords:}

SDNR

Signal-to-noise ratio

Image quality

Quality controls

\begin{abstract}
A B S T R A C T
The standard approach to signal difference-to-noise ratio (SDNR) analysis requires a region of interest (ROI) positioned within the object to measure signal-difference, restricting this metric to flat-topped objects with large, sharply delineated areas. This work develops a generalized expression for SDNR (SDNR $)_{\mathrm{g}}$ calculated from a ROI encompassing the object. Signal power, defined as the deviation of pixel values from the mean background due to the object, is used instead of signal-difference. Comparison was first made by simulating ideal flat-topped discs with sharp edges and diameters between 1 and 80 pixels, into a uniformly noisy background using a known

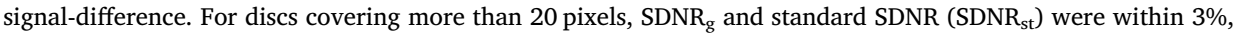
while for discs of less than 20 pixels, SDNR $_{g}$ was within $26 \%$ of the truth compared to $58 \%$ for $S_{D N R}$. Generalized and standard SDNR were compared for radiography images of three different phantoms with microcalcification-like objects (MTM-100 phantom), hemispheric objects of different thicknesses with a Gaussian intensity distribution and mammography quality control (QC) images. Applied to Gaussian details, SDNR $_{\mathrm{g}}$ was between $20 \%$ and $45 \%$ higher than SDNR $_{\text {st }}$, depending on object thickness, while for the QC images, SDNR $_{\mathrm{g}}$ was with $1.7 \%$ of the standard SDNR. Compared to the standard SDNR, SDNR $\mathrm{g}$ is applicable to non-uniform signals, where an explicit contrast measurement is not suitable, and has improved accuracy when assessing SDNR of small objects.
\end{abstract}

\section{Introduction}

Object detection in a uniform noisy background is, to a first approximation, correlated with the signal difference-to-noise ratio (SDNR) for a range of object sizes and shapes, imaging modalities and image characteristics [1-6]. A target SDNR approach has been used for stability controls of image quality [7], to find the minimal dose necessary to achieve the limiting image quality defined in guidelines for a given beam quality and imaging system [8], and to characterize object detectability $[9,10]$.

The standard SDNR analysis relies on object contrast (mean signal difference), which can be measured precisely for a flat-topped signal (object) with sharp edges (perfect resolution) and a sufficiently large area to place a region of interest (ROI) inside [11,12]. The object ROI must be placed within the object thereby avoiding the periphery and any contamination from the surrounding background. This can be difficult to achieve, particularly in small objects composed of a restricted number of pixels/voxels with an irregular outline or shape. Furthermore, for non-flat objects or in the presence of edge effects (edge enhancement, partial volume effects), the spatial distribution of pixel values in the object is not uniform. In this case, the SDNR will use a mean signal-difference that is sensitive to the ROI position and size within the object.

This work presents a generalized approach to SDNR, termed SDNR in which the whole signal power due to the object is considered instead of mean contrast or peak object signal. The signal power is measured from a ROI that encompasses the whole object. The model is validated through measurements made on a synthetic image that satisfy the limiting conditions for standard SDNR measurement, and for radiography images of test objects of different shapes, sizes and contrasts. $\mathrm{SDNR}_{\mathrm{g}}$ is shown to improve the accuracy of the measurement for small objects, and extends the metric to non-uniform objects or objects with edge enhancement. This generalized form of SDNR is also suitable for images with reduced zero-frequency content and objects with strong edge effects, such as the reconstructed planes generated by digital breast tomosynthesis (DBT) systems.

\footnotetext{
* Corresponding author.

E-mail address: pascal.monnin@chuv.ch (P. Monnin).
} 


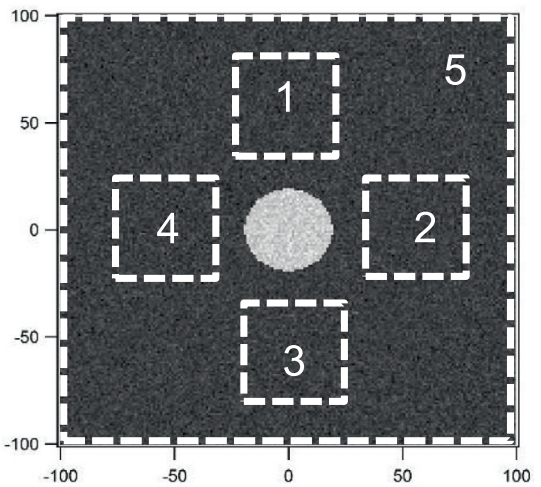

Fig. 1. Synthetic image of a disc in a uniform noisy background with an example of ROIs that can be used for generalized SDNR measurement.

\section{Material and methods}

\subsection{Generalized SDNR}

We consider any object of $N_{o b j}$ pixels (for instance, disc in Fig. 1) in an image with a homogenous noisy background. We define the 'energy' $(W)$ added to a uniform background in a ROI of $N$ pixels as the squared deviation of pixel values from a mean background value $\bar{d}_{b}$.

$$
\begin{aligned}
W & =\sum_{i=1}^{N}\left(d_{i}-\bar{d}_{b}\right)^{2}=\sum_{i=1}^{N} d_{i}^{2}-2 \bar{d}_{b} \sum_{i=1}^{N} d_{i}+\sum_{i=1}^{N} \bar{d}_{b}^{2} \\
& =\sum_{i=1}^{N} d_{i}^{2}-2 N \bar{d}_{b} \bar{d}+N \bar{d}_{b}^{2}
\end{aligned}
$$

This energy can be due to a deterministic signal generated by an object or can come from stochastic fluctuations (noise). The 'power' is the energy normalized by the number of pixels in the ROI: $W / N$. The symbol $\sigma^{2}$ will refer to the power when it is equal to the variance calculated in different ROIs of the image.

The mean background signal $\bar{d}_{b}$ and standard deviation $\sigma_{b}$ are measured in one or several ROIs placed around the object (ROIs 1-4 in Fig. 1). A large ROI of $N$ pixels that encompasses the whole object and a part of the surrounding background (ROI 5 in Fig. 1) generates a mean pixel value $\bar{d}$ (Eq. (2)) and a variance of pixel values $\sigma^{2}$ (Eq. (3)).

$\bar{d}=\frac{1}{N} \sum_{i=1}^{N} d_{i}$

$\sigma^{2}=\frac{1}{N} \sum_{i=1}^{N}\left(d_{i}-\bar{d}\right)^{2}=\left(\frac{1}{N} \sum_{i=1}^{N} d_{i}^{2}\right)-\bar{d}^{2}$

Substituting $\sum_{i=1}^{N} d_{i}^{2}=N\left(\sigma^{2}+\bar{d}^{2}\right)$ from Eq. (3) into Eq. (1):

$W=N\left(\sigma^{2}+\bar{d}^{2}\right)-2 N \bar{d}_{b} \bar{d}+N \bar{d}_{b}^{2}=N\left(\left(\bar{d}-\bar{d}_{b}\right)^{2}+\sigma^{2}\right)$

This energy can be separated into three contributions, related to the powers $P_{s}, \sigma_{n}^{2}$ and $\sigma_{b}^{2}$.

1) signal energy (due to the object):

$W_{s}=N_{o b j} P_{s}$

2) noise energy due to stochastic fluctuations within the object:

$W_{n}=N_{o b j} \sigma_{n}^{2}$

3) noise energy due to stochastic fluctuations outside the object:

$W_{b}=\left(N-N_{o b j}\right) \sigma_{b}^{2}$

$W_{n}$ and $W_{b}$ can be summed because they represent noise energy in different regions of the image (inside and outside the object). $W_{s}$ can be summed with the noise energy because it comes from a deterministic signal added to background noise fluctuations.

$W=W_{s}+W_{n}+W_{b}=N_{o b j} P_{s}+N_{o b j} \sigma_{n}^{2}+\left(N-N_{o b j}\right) \sigma_{b}^{2}$

The signal power $P_{s}$ is obtained from Eqs. (4) and (5).

$P_{s}=\frac{N}{N_{o b j}} \cdot\left(\left(\bar{d}-\bar{d}_{b}\right)^{2}+\sigma^{2}-\sigma_{b}^{2}\right)-\left(\sigma_{n}^{2}-\sigma_{b}^{2}\right)$

A signal larger or smaller than the mean background will give a positive signal power in Eq. (6) because of the difference squared $\left(\bar{d}-\bar{d}_{b}\right)^{2}$. The signal power is therefore sensitive to the amplitude of the difference, not to the sign of this difference. This remark also applies to the standard SDNR, where the absolute signal difference is used. The SDNR is the ratio between the square root of the signal and noise powers.

$S D N R_{g}=\frac{\sqrt{P_{s}}}{\sigma_{b}}=\sqrt{\frac{N}{N_{o b j}} \cdot\left(\left(\frac{\bar{d}-\bar{d}_{b}}{\sigma_{b}}\right)^{2}+\left(\frac{\sigma}{\sigma_{b}}\right)^{2}-1\right)-\left(\left(\frac{\sigma_{n}}{\sigma_{b}}\right)^{2}-1\right)}$

Eq. (7) gives a general formulation of SDNR that encompasses the whole signal power, including signal non-uniformities like edge enhancements or partial volume effects. This is termed the "generalized SDNR $\left(\right.$ SDNR $\left._{\mathrm{g}}\right)$ ".

The practical calculation of Eq. (7) needs to address two points. First, a precise estimation of $N_{o b j}$ used for $\mathrm{SDNR}_{\mathrm{g}}$ may be challenging for images with a low SDNR. Prior knowledge of the object size or adequate object segmentation methods can be used for this purpose. This aspect is discussed in the paragraph 3.5. Secondly, the noise within the object $\left(\sigma_{n}\right)$ is not measurable for non-flat-topped objects $\left(\sigma_{n}^{2}\right.$ will encompass both signal and noise powers), and a reasonable assumption about noise behaviour within the object has to be made. Two typical situations are presented.

Case 1:. Uniform noise in the object equal to background noise $\left(\sigma_{n}=\sigma_{b}\right)$. This case applies to thin objects that hardly influence the noise. Eqs. (6) and (7) revert to Eqs. (8) and (9), respectively.

$P_{s}=\frac{N}{N_{o b j}} \cdot\left(\left(\bar{d}-\bar{d}_{b}\right)^{2}+\sigma^{2}-\sigma_{b}^{2}\right)$

$S D N R_{g}=\sqrt{\frac{N}{N_{o b j}} \cdot\left(\left(\frac{\bar{d}-\bar{d}_{b}}{\sigma_{b}}\right)^{2}+\left(\frac{\sigma}{\sigma_{b}}\right)^{2}-1\right)}$

If formula (9) is used instead of Eq. (7) when noise in the object is different from the background noise, $\mathrm{SDNR}_{\mathrm{g}}$ will deviate from the true value. The error remains very low for most practical situations, and has to be considered only for low SDNR with $\sigma_{n}>>\sigma_{b}$ or $\sigma_{n}<<\sigma_{b}$ (Fig. 2).

Case 2:. Image with a noise variance proportional to the mean pixel value in each point of the image. This case is expected for quantum limited images for $x$-ray projection modalities. For images with pixel values expressed in exposure values, the noise in the object is related to background noise according to Eq. (10).

$\sigma_{n}^{2}=\frac{\bar{d}_{o b j}}{\bar{d}_{b}} \sigma_{b}^{2}=\left(1+\frac{N}{N_{o b j}}\left(\frac{\bar{d}}{\bar{d}_{b}}-1\right)\right) \sigma_{b}^{2}$

where $\bar{d}_{o b j}$ is the mean pixel value in the object.

$\bar{d}_{o b j}=\bar{d}_{b}+\frac{N}{N_{o b j}}\left(\bar{d}-\bar{d}_{b}\right)$

Eqs. (6), (7) and (10) give:

$P_{s}=\frac{N}{N_{o b j}} \cdot\left(\left(\bar{d}-\bar{d}_{b}\right)^{2}+\sigma^{2}-\frac{\bar{d}}{\bar{d}_{b}} \sigma_{b}^{2}\right)$

$S D N R_{g}=\sqrt{\frac{N}{N_{o b j}} \cdot\left(\left(\frac{\bar{d}-\bar{d}_{b}}{\sigma_{b}}\right)^{2}+\left(\frac{\sigma}{\sigma_{b}}\right)^{2}-\frac{\bar{d}}{\bar{d}_{b}}\right)}$ 


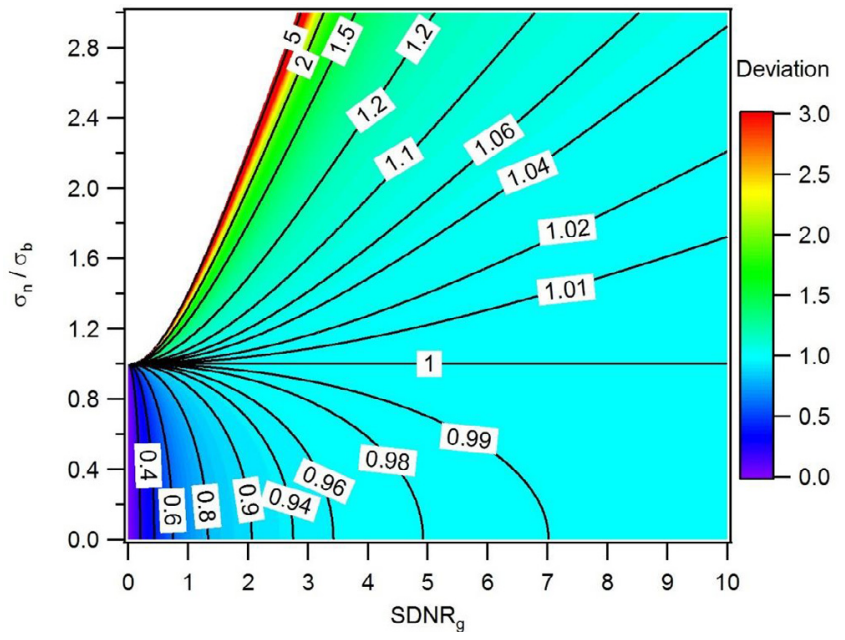

Fig. 2. Deviation from the true value of $\mathrm{SDNR}_{\mathrm{g}}$ (colour scale) as a function of the ratio $\sigma_{n} / \sigma_{b}$ if Eq. (9) is used instead of Eq. (7) (ratio Eqs. (9)/(7)).

In the following sections, Eq. (13) is applied to small objects in the form of microcalcifications and to extended non-uniform objects.

\subsection{Link between the generalized and standard SDNR}

This section investigates the differences between the generalized and standard SDNR ( $\mathrm{SDNR}_{\mathrm{st}}$ ). SDNR $\mathrm{st}$ can be expressed using Eq. (11) for $\bar{d}_{o b j}$.

$S D N R_{s t}=\frac{\left|\bar{d}_{o b j}-\bar{d}_{b}\right|}{\sigma_{b}}=\frac{N}{N_{o b j}} \cdot \frac{\left|\bar{d}-\bar{d}_{b}\right|}{\sigma_{b}}$

By definition, signal power and SDNR should be independent of the ratio $N / N_{o b j}$. The large ROI of smallest possible size follows the boundary of the object, with $N=N_{o b j}$ and $\bar{d}=\bar{d}_{o b j}$. As a consequence, Eqs. (6) and (7) are equal to Eqs. (15) and (16), respectively.

$P_{s}=\left(\bar{d}_{o b j}-\bar{d}_{b}\right)^{2}+\sigma_{\mid N=N_{o b j}}^{2}-\sigma_{n}^{2}$

$S D N R_{g}=\sqrt{\left(\frac{\bar{d}_{o b j}-\bar{d}_{b}}{\sigma_{b}}\right)^{2}+\frac{\sigma_{\mid N=N_{o b j}}^{2}-\sigma_{n}^{2}}{\sigma_{b}^{2}}}$

The signal power depends on the object contrast $\left|\bar{d}_{o b j}-\bar{d}_{b}\right|$ and on the distribution of pixel values within the object $\left(\sigma_{\mid N=N_{o b j}}^{2}\right)$ :

$\left\{\begin{array}{c}\sigma_{\mid N=N_{o b j}=\sigma_{n}^{2}} \text { for a flat }- \text { topped (uniform) object } \\ \sigma_{\mid N=N_{o b j}}^{2}>\sigma_{n}^{2} \text { otherwise }\end{array}\right.$

$\sqrt{P_{S}}$ is therefore greater or equal to the object contrast (Eq. (18)), and $\mathrm{SDNR}_{\mathrm{g}}$ is greater or equal to $\mathrm{SDNR}_{\text {st }}$ (Eq. (19)), depending on the object characteristics.

$\sqrt{P_{s}} \geq\left|\bar{d}_{o b j}-\bar{d}_{b}\right|$

$S D N R_{g} \geq S D N R_{S t}$

The validity of the standard SDNR is restricted to flat-topped objects with sharply delineated areas in uniform noisy backgrounds. For this particular case, $\sqrt{P_{s}}$ and object contrast are equal and the generalized SDNR (Eq. (16)) reverts to the standard SDNR (Eq. (14)).

\subsection{SDNR assessment}

The accuracy and robustness of the proposed generalized SDNR were tested and compared to the standard SDNR with a set of experiments.

\subsubsection{Ideal case of flat discs}

The behaviour of the generalized and standard SDNR for the ideal condition of a sharp-edged flat object in a uniform noisy background was studied for synthetic images of discs of various radii between 1 and 80 pixels (areas between $\sim 3$ and 20,106 pixels) within an image of $N=200 \times 200$ pixels ( $N$ constant in all simulations). Fig. 1 shows an example for a disc of 1000 pixels. The background has a mean pixel value $\bar{d}_{b}=100$ and a Gaussian noise of variance $\sigma_{b}^{2}=100$. The discs, centred in the image, have a mean pixel value $\bar{d}_{o b j}=200$ and a Gaussian noise of variance $\sigma_{n}^{2}=200$. The discs have sharp edges, and no blur was applied to the images, giving a resolution limited by the pixel size. SDNR $_{\text {g }}$ (Eq. (7)) and SDNR st (Eq. (14)) were computed for 100 different statistically independent realizations of Gaussian noise for each disc size. $\bar{d}$ and $\sigma$ were calculated from the whole images. A Boolean subtraction of the disc in the images gave $\bar{d}_{b}, \bar{d}_{o b j}, \sigma_{b}$ and $\sigma_{n}$. The average value and standard deviation of the 100 SDNR realizations gave information on the accuracy and variability of the standard and generalized SDNR as a function of the object size.

\subsubsection{Application to small objects}

A mammography MTM-100 phantom (Medi-Test, France) was imaged on a Hologic Selenia Dimensions system at $28 \mathrm{kV}$ and $\mathrm{W} / \mathrm{Rh}$ anode/filter combination at five exposure levels (117, 156, 202, 248 and $308 \mathrm{mAs}$ ). The SDNR for the first group of microcalcifications (six spheres of diameter $0.3 \mathrm{~mm}$ ) were calculated from the pre-processed ('For Processing') and processed ('For Presentation') images. SDNR $\mathrm{g}$ was calculated using Eq. (13). The large ROI included the six microcalcifications and $N_{o b j}$ was estimated from the known object size and pixel size $(70 \mu \mathrm{m})$. The background ROI was positioned between the microcalcifications. The peak signal of the microcalcifications was used instead of $\bar{d}_{o b j}$ for the standard SDNR, as it is usually done when the object covers a few number of pixels.

\subsubsection{Application to non-uniform objects}

$\mathrm{SDNR}_{\mathrm{g}}$ to $\mathrm{SDNR}_{\mathrm{st}}$ were compared for (non-flat) objects of different shapes and contrasts. A test object made of a $20 \mathrm{~mm}$ thick PMMA plate containing five hemispheric objects with a Gaussian shape varying between 1 and $3 \mathrm{~mm}$ in steps of $0.5 \mathrm{~mm}$ was imaged on the flat panel detector of a Siemens FLC Compact general radiography system $(60 \mathrm{kV}$ - $1.7 \mathrm{mAs}$ - focus-to-detector distance $1.2 \mathrm{~m}$ ). The 12-bit 'For Processing' DICOM image was then processed with an edge enhancement filter and a low-pass filter, giving five objects on three images with different processing. $\bar{d}_{b}$ and $\sigma_{b}$ were measured in four $10 \times 10 \mathrm{~mm}$ ROIs placed next to the four sides of the objects, and averaged (ROIs like in Fig. 1). ROIs of $8 \times 8 \mathrm{~mm}$ placed within each object gave $\bar{d}_{o b j}$ for SDNR $_{\text {st. }}$ A large $20 \times 20 \mathrm{~mm}$ ROI including the object gave $\bar{d}$ and $\sigma$ for $\mathrm{SDNR}_{\mathrm{g}}$, calculated with Eq. (13). $N_{o b j}$ was estimated from the known objects diameter $(12 \mathrm{~mm})$, pixel size $(139 \mu \mathrm{m})$ and geometrical magnification due to the $\mathrm{x}$-ray beam divergence $(1 \%)$.

\subsubsection{Application to $Q C$ test images}

SDNR $_{g}$ obtained with Eqs. (7) and (9) were compared to SDNR $_{\text {st }}$ for images used for quality controls (QC) in mammography (European Guidelines 2006). A $50 \mathrm{~mm}$ PMMA thickness with a $10 \times 10 \times 0.2 \mathrm{~mm}$ aluminium square sandwiched at a height of $20 \mathrm{~mm}$ and placed at $60 \mathrm{~mm}$ from the chest wall was imaged using the AEC (auto-filter) mode on a Hologic Selenia Dimensions system $(31 \mathrm{kV}-151 \mathrm{mAs}-\mathrm{W} / \mathrm{Rh})$. The 12-bit 'For Processing' DICOM image was used. $\bar{d}_{b}$ and $\sigma_{b}$ were measured in four $10 \times 10 \mathrm{~mm}$ ROIs placed next to the four sides of the aluminium square, and averaged (ROIs like in Fig. 1). A $8 \times 8 \mathrm{~mm}$ ROI placed within the $\mathrm{Al}$ square gave $\bar{d}_{o b j}$ for $\mathrm{SDNR}_{\mathrm{st}}$ and $\sigma_{n}$ for $\mathrm{SDNR}_{\mathrm{g}}$. A large $20 \times 20 \mathrm{~mm}$ ROI including the $\mathrm{Al}$ square gave $\bar{d}$ and $\sigma$ for SDNR $_{\mathrm{g}}$. The object was delimited in the image with a threshold pixel value to estimate $N_{o b j}$. 


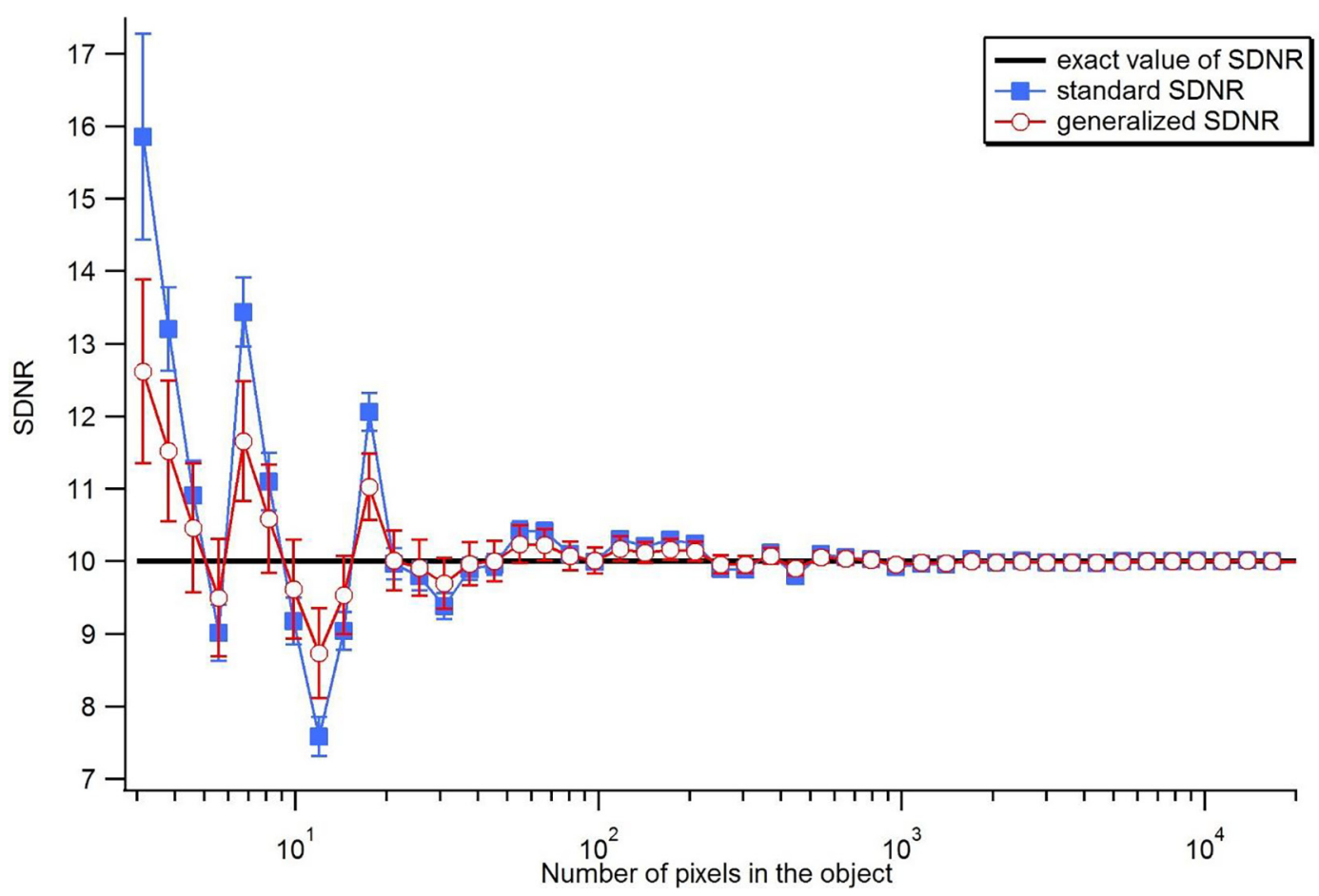

Fig. 3. Mean values and standard deviation (error bars) of $\mathrm{SDNR}_{\mathrm{st}}$ and $\mathrm{SDNR}_{\mathrm{g}}$ as a function of the object size, obtained over 100 random realizations of noise.

\section{Results and discussion}

\subsection{Ideal case of flat discs}

The known true SDNR value for all the disc sizes is equal to 10 , illustrated by the line in Fig. 3. When the object is sufficiently large, statistical uncertainty in the calculated variances becomes small enough to show the invariance of $\mathrm{SDNR}_{\mathrm{g}}$ regarding the ratio $N / N_{o b j}$ (Fig. 3). Considering discs of more than 20 pixels, $\mathrm{SDNR}_{\mathrm{g}}$ and $\mathrm{SDNR}_{\mathrm{st}}$ agreed within $3 \%$, while for discs of less than 20 pixels, SDNR $_{\mathrm{g}}$ was within $26 \%$ of the true value compared to $58 \%$ for SDNR $_{\text {st }}$. The standard and generalized SDNR show the same oscillations around the true SDNR value for small discs, with an accuracy increasing with the object area. These oscillations are important only for objects covering a few pixels. The number of pixels attributed to the object or background (as a function of their value) varies with visible thresholds when the object area varies around a small number of pixels. As a consequence, the statistical values in the ROIs alternatively give under- and overestimations around the true value when the object size grows from a very small number of pixels. The amplitude of the oscillations decreases with increasing object area. $\mathrm{SDNR}_{\mathrm{g}}$ is more accurate than $\mathrm{SDNR}_{\mathrm{st}}$ for small objects. The mean object signal used for the standard SDNR is more sensitive to random fluctuations of noise in the object than the signal power calculated over the large ROI including the object. It is of note this calculation does not include the random positioning of the object ROI needed for the standard SDNR, which is an additional source of variability for small objects. The generalized SDNR is less operator dependent, and gives a more robust and accurate evaluation of signal power and SDNR for small objects.

\subsection{Application to small objects}

The standard and generalized SDNR for the microcalcifications in the mammography (MTM-100) phantom increase as a power function of the exposure level (mAs), for both raw and processed images (linear log-log fitted curves in Fig. 4), with $\mathrm{SDNR}_{\text {st }}$ greater than $\mathrm{SDNR}_{\mathrm{g}}$. This result is expected for quantum limited images. Image processing

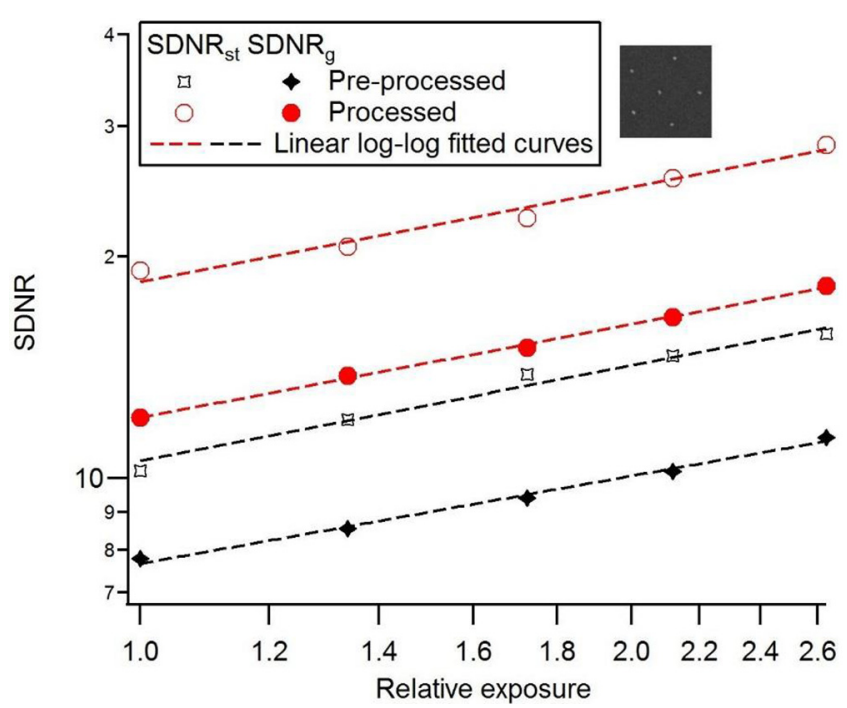

Fig. 4. Standard and generalized SDNR of microcalcifications measured on preprocessed and processed mammography images for different exposure levels.

decreases the background noise and increases the signal power with edge enhancement, thus increases SDNR. In this particular case, the standard SDNR overestimates the signal power because the peak value is used for the object signal.

\subsection{Application to non-uniform objects}

As expected, the standard and generalized SDNR increase with the contrast of the Gaussian objects, and increase/decrease when correlation between neighbouring pixel values increases/decreases due to image filtering (Fig. 5). Here, SDNR $_{\mathrm{g}}$ is systematically higher than $\mathrm{SDNR}_{\text {st }}$, as expected for non-uniform objects (Eq. (18)). The difference, between $20 \%$ and $35 \%$ for the five Gaussian objects on the pre-processed image, is higher for the thicker objects, and is higher (between $30 \%$ and $45 \%$ ) for the image with edge enhancement compared to the 


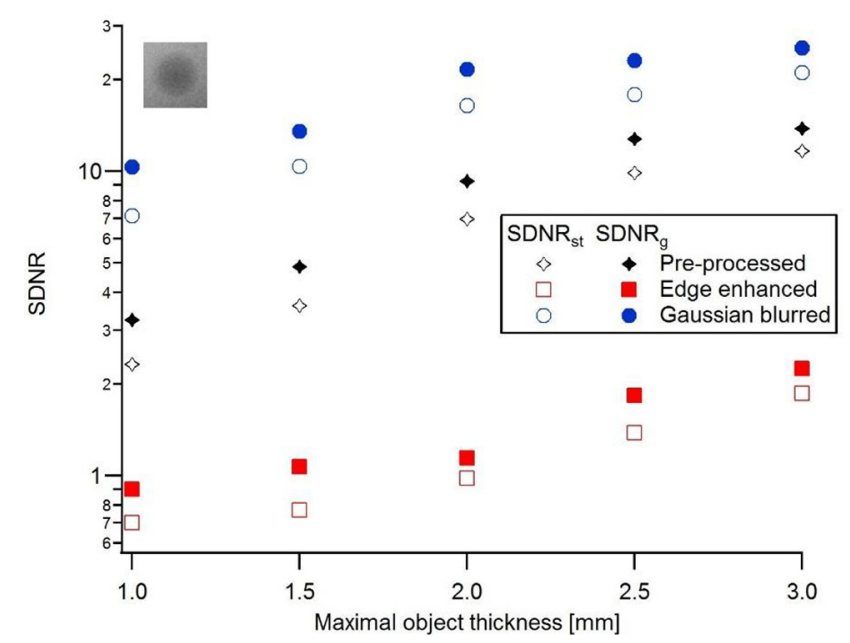

Fig. 5. Standard and generalized SDNR measured on radiography images of Gaussian objects of different contrasts.

Table 1

Statistical values measured in the ROIs in the mammography QC image.

\begin{tabular}{lll}
\hline ROI & Mean pixel value & Std Dev \\
\hline Background & $\bar{d}_{b}=436.81$ & $\sigma_{b}=6.44$ \\
Object & $\bar{d}_{o b j}=390.95$ & $\sigma_{n}=6.05$ \\
Background + Object & $\bar{d}=424.55$ & $\sigma=21.19$ \\
\hline
\end{tabular}

two other images (pre-processed and blurred). The difference between $\mathrm{SDNR}_{\mathrm{g}}$ and $\mathrm{SDNR}_{\mathrm{st}}$ increases when the object shape moves away from the ideal case of a flat-topped object. The difference is higher for the edge enhanced images because $\mathrm{SDNR}_{\mathrm{g}}$ includes the additional signal power at the object perimeter due to the edge enhancement processing, whereas SDNR $_{\mathrm{st}}$ is based only on the mean signal inside the object. There was a difference of just $1 \%$ between formulations (9) and (13) for $\mathrm{SDNR}_{\mathrm{g}}$ (data not shown), calculated on linearized images with pixel values expressed in exposure values, showing the approximation $\sigma_{n} \approx \sigma_{b}$ is suitable for projection images with usual SDNR values (Fig. 2).

\subsection{Application to $Q C$ test images}

The values measured in the three ROIs in the QC mammography image are reported in Table 1 . Eq. (14) gave $S D N R_{s t}=7.12$. With $N=87600$ pixels and $N_{o b j}=24024$ pixels in the large (background + object) ROI, Eqs. (7) and (9) gave $\mathrm{SDNR}_{\mathrm{g}}$ equal to 7.10 and 7.00 , respectively. The difference of $6.5 \%$ between the noise in the object and in its surrounding background is the source of the $1.4 \%$ difference between these two estimations of SDNR . The standard and generalized SDNR are expected to be equal for this kind of (flat) object with sharp edges. The maximal difference of $1.7 \%$ between SDNR $_{\text {st }}$ and $\mathrm{SDNR}_{\mathrm{g}}$ remains at an acceptable level for QC applications.

\subsection{Limitations of the model}

Both the standard and generalized SDNR are first order metrics, with the same inherent limitations due to mathematical assumptions in the model. These metrics need spatial stationarity of signal and noise within the ROIs used for the estimation of the mean and variance of pixel values, and give no information on the spatial frequency content of signal and noise, which is important for object detectability [13]. If stationarity of signal or noise is not met in the ROIs, the variance of pixels values will increase dramatically and jeopardize the SDNR measure. This effect tends to increase with ROI size [14], and therefore the use of reasonably small ROIs is recommended for radiography images. When using Eq. (9) for generalized SDNR, one has to ensure the noise within the object is not significantly different from the background noise, otherwise the generalized SDNR can deviate from the true value. In this case, the more general Eq. (7) has to be used with a direct measurement of noise within the object (for objects with a flat signal on the image), or with a suitable estimation or model for object noise $\left(\sigma_{n}\right)$. The error remains very low for most practical situations, and has to be considered only for low SDNR with $\sigma_{n}>>\sigma_{b}$ or $\sigma_{n}<<\sigma_{b}$ (Fig. 2).

A precise estimation of $N_{o b j}$ needed in $\mathrm{SDNR}_{\mathrm{g}}$ may be difficult for images with a low SDNR. Several methods may be considered for this purpose. The object size on the image may be calculated from the real (known) object size, corrected for geometrical factors such as magnification, or delimited on an image acquired with a high SDNR. In the case of unclear object boundaries (e.g. in low SDNR image), object segmentation based on intensity threshold or gradient methods can be considered. Automated $\mathrm{SDNR}_{\mathrm{g}}$ calculations can be obtained by sorting the pixels belonging to the background and to the object itself.

Final remarks concern the validation of $\mathrm{SDNR}_{\mathrm{g}}$ using human observers. Standard SDNR can be linked to human detectability performance using the Rose model $[11,15]$, for simple targets in uniform noise. We would expect that the generalized SDNR can be used in the Rose model in place of $\mathrm{SDNR}_{\mathrm{st}}$, for example to include the effect of edge enhancements on detectability. The generalized SDNR takes the whole signal power into account, to which the observer will be sensitive (i.e. the observer may accumulate as 'signal'), while the standard SDNR quantifies only the mean signal-background difference. The comparison between $\mathrm{SDNR}_{\mathrm{g}}, \mathrm{SDNR}_{\mathrm{st}}$ and human reader detection performance for a range of targets, including small details and non-uniform or structured targets, requires rigorous observer studies; these are the topic of future study.

\section{Conclusion}

This work has introduced and tested a new generalized methodology for assessing the SDNR that accounts for the total signal power generated by an object in an image, independent of object shape and edge characteristics. The one proviso of the new method is an estimate of the number of pixels within the object of interest. The equivalence to standard SDNR analysis based on object contrast was demonstrated for the ideal case of a flat object in an image with perfect spatial resolution. Compared to the standard SDNR, the generalized SDNR gives a more accurate estimate for small objects, non-flat objects and/or objects with non-sharp edges (blur, partial volume effects, edge enhancement). Moreover, the generalized method removes the variability associated with ROI positioning and size within the object. The generalized SDNR is also more robust in the presence of small structures characterized by low signal statistics where the standard SDNR approach often just uses the peak signal. The generalized formulation is proposed as an alternative to the standard SDNR method and is expected to improve the reliability of the SDNR based methods often used in quality control protocols.

\section{References}

[1] Judy PF, Swenson RG. Lesion detection and signal-to-noise ratio in CT images. Med Phys 1981;8:13-23.

[2] Barrett HH, Rolland JP, Wagner RF, Myers KJ. Detection and discrimination of know signals in homogeneous, random background. Proc SPIE 1990;1090:176-82.

[3] Samei E, Dobbins JT, Lo JY, Tornai MP. A framework for optimizing the radiographic technique in digital X-ray imaging. Radiat Prot Dosim 2005;114:220-9.

[4] Bernhardt P, Mertelmeier T, Hoheisel M. X-ray spectrum optimization of full-field digital mammography: simulation and phantom study. Med Phys 2006;33:4337-43.

[5] Williams MB, Raghunathan P, More MJ, Seibert JA, Kwan A, Lo JY, et al. Optimization of exposure parameters in full field digital mammography. Med Phys 2008;35:2414-23.

[6] Verdun FR, Racine D, Ott JG, Tapiovaara MJ, Toroi P, Bochud FO, et al. Image 
quality in CT: from physical measurements to model observers. Phys Med 2015;31:823-43.

[7] European Guidelines for Breast Cancer Screening. The European protocol for the quality control of the physical and technical aspects of mammography screening. Part B: Digital mammography. 4th ed. Luxembourg: European Commission; 2006.

[8] Young KC, Cook JJH, Oduko JM. Use of the European protocol to optimise a digital mammography system. LNCS 2006;4046:362-9.

[9] Karunamuni R, Tsourkas A, Maidment ADA. Exploring silver as a contrast agent for contrast-enhanced dual-energy X-ray breast imaging. Brit J Radiol 2014;87:20140081.

[10] Salvagnini E, Bosmans H, Struelens L, Marshall NW. Tailoring automatic exposure control toward constant detectability in digital mammography. Med Phys
2015;42:3834-47.

[11] Wagner RF, Brown DG. Unified SNR analysis of medical imaging systems. Phys Med Biol 1985;30:489-518.

[12] Burgess AE. The rose model, revisited. J Opt Soc Am 1999;16:633-46.

[13] ICRU Report 54. Medical imaging - the assessment of image quality. J ICRU os 28. https://doi.org/10.1093/jicru/os28.1.Report54; 1996.

[14] Alsager A, Young KC, Oduko JM. Impact of heel effect and ROI size on the determination of contrast-to-noise ratio for digital mammography systems. Proc SPIE 2008;6913. 691341-1-11.

[15] Marshall NW. A comparison between objective and subjective image quality measurements for a full field digital mammography system. Phys Med Biol 2006;51:2441-63. 